J. Venom. Anim. Toxins incl. Trop. Dis.

V.11, n.2, p.214, 2005.

Thesis - ISSN 1678-9199.

\title{
EVALUATION OF Histoplasma capsulatum INFECTION IN INHABITANTS OF THE URBAN AND RURAL AREAS OF PRATÂNIA, SÃO PAULO, BY MEANS OF INTRADERMAL REACTIONS
}

THESIS. C. C. Rodrigues submitted this thesis for her Doctorate in Tropical Diseases at Botucatu School of Medicine, São Paulo State University, UNESP, Botucatu, São Paulo, Brazil, 2004.

Advisor: Professor Rinaldo Poncio Mendes

ABSTRACT: The presence of various pathogenic fungi in healthy hosts, as can be shown by skin tests of randomized populations, has attracted the attention of the scientific community and has a great significance on the increasing number of immunosuppressed individuals. Skin tests with histoplasmin and paracoccidioidin were performed with 338 individuals from the urban area and 78 from a poultry farm, both in the town of Pratânia - São Paulo State (Brazil), in order to detect the prevalence of histoplasmosis and paracoccidioidomycosis infection. The histoplasmin utilized was the sterile culture filtrate obtained from the mycelial growth of the B-679 and A-811 strains of Histoplasma capsulatum var. capsulatum; the paracoccidioidin utilized was the sterile polysaccharide antingen prepared from a pool of several Paracoccidioides brasiliensis strains. The tests were carried out by injecting $0.1 \mathrm{ml}$ of the dilution (1:1000 for histoplasmin and 1:10 for paracoccidioidin) by the intradermal route into the anterior surface of each individual's forearm between November and December 2003. Readings of the reaction were taken 24 and 48 hours after inoculation. The tests were considered positive when papules with a diameter of $5 \mathrm{~mm}$ or more were observed after 24 or 48 hours. An individual was considered infected by $H$. capsulatum or $P$. brasiliensis when his/her skin test was positive to only one antigen. Data were statistically analyzed by the chi-square, Fisher exact, McNemar, Wilcoxon, $t$-Student, and Goodman tests, with significance level set at $5 \%$. The concordance correlation test was utilized too. The 
prevalence of histoplasmosis infection in the urban area was $13.0 \%$ and paracoccidioidomycosis infection, 51.2\%. A relationship between the positivity of the skin tests and the different variables studied (i.e., distribution, gender, habits, period of residence in this town, and activities developed by the inhabitants) was not observed. Relationship between the positivity of skin tests to histoplasmin and the contact with excrements from chickens and bats was not demonstrated either. All the inhabitants of the poultry farm with positive skin tests to histoplasmin were also positive to paracoccidiodin. The study of 40 children from the urban area, aged from 5 to 13 years showed $2.5 \%$ of positivity to histoplasmin and $32.5 \%$ to paracoccidioidin. The prevalence of positive skin tests was higher in the 48-hours reading for histoplasmin and in the 24-hours reading for paracoccidioidin; however, the average and the median of the 24-hours and 48-hours readings were not different for histoplamin and for paracoccidioidin, respectively. Finally, the degree of concordance between the 24-hours and the 48-hours readings was good (kappa $=0.61$ ) for the histoplasmin test and very good (kappa $=0.85)$ for the paracoccidioidin test. The results show that the town of Pratânia is an endemic area of histoplasmosis and a highly endemic area of paracoccidioidomycosis, and reveal an early infection by $P$. brasiliensis.

KEY WORDS: Histoplasma capsulatum, histoplasmin, histoplasmosis.

CORRESPONDENCE TO: C. C. RODRIGUES, Departamento de Doenças Tropicais e Diagnóstico por Imagem, Faculdade de Medicina de Botucatu, UNESP, Distrito de Rubião Junior, s/n, 18618-000, Botucatu, SP, Brasil. Phone: 551438116212.

Email: rmendes@fmb.unesp.br 